\title{
QUADRATIC SPACES OVER LAURENT EXTENSIONS OF DEDEKIND DOMAINS
}

BY

\author{
RAMAN PARIMALA
}

\begin{abstract}
Let $R$ be a Dedekind domain in which 2 is invertible. We show in this paper that any isotropic quadratic space over $R\left[T, T^{-1}\right]$ is isometric to $q_{1} \perp T q_{2}$ where $q_{1}, q_{2}$ are quadratic spaces over $R$. We give an example to show that this result does not hold for anisotropic spaces.
\end{abstract}

Introduction. Let $R$ be a Dedekind domain in which 2 is invertible. Karoubi, in [3], shows that if $q$ is any quadratic space over $R\left[T, T^{-1}\right]$, then, the class [ $\left.q\right]$ of $q$ in the Wittring of $R\left[T, T^{-1}\right]$ is $\left[q_{1}\right]+\left[T q_{2}\right]$, where $q_{1}$ and $q_{2}$ are quadratic spaces over $R$. In this paper, we prove a nonstable version of this theorem.

In $\S 1$, we list a few results on quadratic spaces over $K\left[T, T^{-1}\right], K$ denoting a field of characteristic $\neq 2$ and also recall the stable results of Karoubi. In $\S 2$, we show (Theorem 2.4) that if $R$ is a complete discrete valuation ring in which 2 is invertible, any quadratic space over $R\left[T, T^{-1}\right]$ is isometric to $q_{1} \perp T q_{2}, q_{1}$ and $q_{2}$ being quadratic spaces over $R$. This shows in particular that any quadratic space over $R\left[T, T^{-1}\right]$ is an orthogonal sum of rank one spaces. If $R$ is a field, this result is due to Harder (see [4, 13.4.4]). In $\$ 3$, using the technique of patching diagrams, we prove the main result (Theorem 3.5), namely, if $R$ is a Dedekind domain in which 2 is invertible, every isotropic quadratic space over $R\left[T, T^{-1}\right]$ is isometric to $q_{1} \perp T q_{2}$ where $q_{1}$ and $q_{2}$ are quadratic spaces over $R$. We give an example to show that this result does not hold for anisotropic spaces.

In this paper, $R$ denotes an integral domain in which 2 is invertible. A quadratic space $(P, q)$ over $R$ is denoted by $q$, suppressing the underlying module. We say that a quadratic space $(P, q)$ is isotropic if there exists $v \in P, v \neq 0$ such that $q(v)=0$. For $\lambda_{i} \in U(R), 1 \leqslant i \leqslant n,\left\langle\lambda_{1}, \ldots, \lambda_{n}\right\rangle$ denotes the diagonal form. We denote by $h$ the standard hyperbolic plane over $R$ whose matrix is $\left(\begin{array}{ll}0 & 1 \\ 1 & 0\end{array}\right)$. The Witt index of $a$ quadratic space $q$ over $R$ is $\geqslant n$ if $q \simeq q^{\prime} \perp H(P)$, with rank $P \geqslant n$, where $H(P)$ denotes the hyperbolic space.

1. Some preliminary results. In this section we prove some lemmas which will be used in the later sections. We assume throughout the paper that in all the rings considered, 2 is invertible. If $q_{1}, q_{2}$ are quadratic spaces over $R, q_{1} \perp T q_{2}$ denotes the quadratic space $\left(q_{1} \otimes_{R} R\left[T, T^{-1}\right]\right) \perp T\left(q_{2} \otimes_{R} R\left[T, T^{-1}\right]\right)$ over $R\left[T, T^{-1}\right]$.

Received by the editors February 16, 1982.

1980 Mathematics Subject Classification. Primary 13D15, 10C05, 15A63; Secondary 13F05.

Key words and phrases. Quadratic spaces, isotropy, Laurent-extensions, Dedekind domains. 
LEMMA 1.1 Let $R$ be an integral domain. Let $q_{1}$ and $q_{2}$ be quadratic spaces over $R$. If the quadratic space $q_{1} \perp T q_{2}$ over $R\left[T, T^{-1}\right]$ is isotropic, then $q_{1}$ or $q_{2}$ is isotropic over $R$.

Proof. Clearly it suffices to prove the lemma when $R$ is a field. Let $q_{1} \simeq$ $\left\langle\lambda_{1}, \ldots, \lambda_{r}\right\rangle, q_{2} \simeq\left\langle\mu_{1}, \ldots, \mu_{s}\right\rangle, \lambda_{i}, \mu_{i} \in R-(0)$. If $q_{1} \perp T q_{2}$ is isotropic, there exist $f_{i}, g_{j} \in R[T], 1 \leqslant i \leqslant r, 1 \leqslant j \leqslant s$, such that $\Sigma \lambda_{i} f_{i}^{2}+T \cdot \Sigma \mu_{j} g_{j}^{2}=0$. Let $n=$ $\max _{i}\left\{\right.$ degree $\left.f_{i}\right\}$ and $m=\max _{j}\left\{\right.$ degree $\left.g_{j}\right\}$. Let $\theta_{i}$ (resp. $\varphi_{j}$ ) be the coefficients of $T^{n}$ (resp. $T^{m}$ ) in $f_{i}$ (resp. $g_{j}$ ). A comparison of degree shows that $\Sigma \lambda_{i} \theta_{i}^{2}=0$ or $\Sigma \mu_{j} \varphi_{j}^{2}=0$ i.e. $q_{1}$ or $q_{2}$ is isotropic over $R$.

LEMMA 1.2. Let $K$ be a field of characteristic $\neq 2$. Any quadratic space over $K\left[T, T^{-1}\right]$ is isometric to $q_{1} \perp T q_{2} \perp h^{n}$ where $q_{1}$ and $q_{2}$ are quadratic spaces over $K$ and $n=$ Witt index of $q$.

Proof. Since $K\left[T, T^{-1}\right]$ is Euclidean, $q \simeq q^{\prime} \perp h^{n}$, where $q^{\prime}$ is anisotropic and $n=$ Witt index of $q$. Since Spec $K\left[T, T^{-1}\right]$ is the complement in $\mathbf{P}_{1}(K)$ of two points, each of degree one, it follows from $[4,13.4 .4]$ that $q^{\prime}$ is diagonalisable. Hence $q^{\prime} \simeq q_{1} \perp T q_{2}, q_{1}, q_{2}$ quadratic spaces over $K$.

LEMMA 1.3. Let $K$ be a field of characteristic $\neq 2$. Then cancellation holds for quadratic spaces over $K\left[T, T^{-1}\right]$.

Proof. Let $q, q^{\prime}, q^{\prime \prime}$ be quadratic spaces over $K\left[T, T^{-1}\right]$ such that $q \perp q^{\prime \prime} \simeq q^{\prime} \perp$ $q^{\prime \prime}$. If $q$ is isotropic, in view of cancellation for isotropic quadratic spaces over principal ideal domains [14, Theorem 3.1], $q \simeq q^{\prime}$. Let $q$ be anisotropic. Then $q^{\prime}$ is also anisotropic and by Lemmas 1.2 and 1.1, $q \simeq q_{1} \perp T q_{2}, q^{\prime} \simeq q_{1}^{\prime} \perp T q_{2}^{\prime}$ where $q_{i}$, $q_{i}^{\prime}, i=1,2$, are anisotropic quadratic spaces over $K$. Let $q_{1} \simeq\left\langle\lambda_{1}, \ldots, \lambda_{r}\right\rangle, q_{2} \simeq$ $\left\langle\mu_{1}, \ldots, \mu_{s}\right\rangle, q_{1}^{\prime} \simeq\left\langle\lambda_{1}^{\prime}, \ldots, \lambda_{l}^{\prime}\right\rangle, q_{2}^{\prime} \simeq\left\langle\mu_{1}^{\prime}, \ldots, \mu_{k}^{\prime}\right\rangle, \lambda_{i}, \mu_{i}, \lambda_{i}^{\prime}, \mu_{i}^{\prime} \in K^{*}$. Over $K(T)$, $q_{1} \perp T q_{2} \simeq q_{1}^{\prime} \perp T q_{2}^{\prime}$ and hence $q_{1}^{\prime} \perp T q_{2}^{\prime}$ represents $\lambda_{1}$ over $K(T)$. Thus, there exist $f_{i}, g_{i}, g \in K[T]$ such that

$$
\lambda_{1} g^{2}=\sum_{i=1}^{l} \lambda_{i}^{\prime} f_{i}^{2}+T \sum_{j=1}^{k} \mu_{j}^{\prime} g_{j}^{2} .
$$

Since the polynomials $\Sigma \lambda_{i}^{\prime} f_{i}^{2}$ and $\Sigma \mu_{j}^{\prime} g_{j}^{2}$ have even degrees, $q_{1}^{\prime}$ and $q_{2}^{\prime}$ being anisotropic, a comparison of degrees in $(*)$ shows that degree $\Sigma \lambda_{i}^{\prime} f_{i}^{2}>$ degree $T \cdot \Sigma \mu_{j}^{\prime} g_{j}^{2}$. Comparing the leading coefficients in $(*)$, it follows that $q_{1}^{\prime}$ represents $\lambda_{1}$. By induction, one shows that $q_{1} \simeq q_{1}^{\prime}$ and $q_{2} \simeq q_{2}^{\prime}$.

Let now $R$ be a Dedekind domain. The canonical map $R \hookrightarrow R\left[T, T^{-1}\right]$ induces an isomorphism Pic $R \stackrel{\sim}{\rightarrow} \operatorname{Pic} R\left[T, T^{-1}\right]$. In what follows, we shall regard this as an identification.

LEMMA 1.4. Let $R$ be a Dedekind domain and $q$ a quadratic space over $R\left[T, T^{-1}\right]=A$. Then there exist quadratic spaces $q_{1}$ and $q_{2}$ over $R$ such that $q \perp h^{m} \simeq q_{1} \perp T q_{2} \perp$ $h^{m-1} \perp H(Q)$ for some $Q \in \operatorname{Pic} R$.

Proof. By a theorem of Karoubi [3, Theorem 3.11], the class [ $q]$ of $q$ in the Wittring of $A$ is equal to $\left[q_{1}\right]+\left[T q_{2}\right], q_{1}, q_{2}$ being quadratic spaces over $R$. Hence 
$q \perp h^{m} \simeq q_{1} \perp T q_{2} \perp H(P)$ for some projective $A$-module $P$. We assume without loss of generality that $q_{1}$ and $q_{2}$ are anisotropic since over any Dedekind domain, any isotropic space splits off a hyperbolic summand. Hence, by Lemma 1.1, $q_{1} \perp T q_{2}$ is anisotropic. Comparing the Witt indices of $q \perp h^{m}$ and $q_{1} \perp T q_{2} \perp H(P)$ over $K\left[T, T^{-1}\right], K$ denoting the quotient field of $R$, it follows that rank $P \geqslant m$. Hence $H(P) \simeq h^{m-1} \perp H(Q) \perp h^{r}$ where $r+m=\operatorname{rank} P$ and $Q=\wedge^{r+m} P \in$ Pic $R$. If $q_{1}^{\prime}=q_{1} \perp h^{r}$, then $q \perp h^{m} \simeq q_{1}^{\prime} \perp T q_{2} \perp h^{m-1} \perp H(Q)$.

COROLlary 1.5. Let $R$ be a Dedekind domain and $q$ an isotropic quadratic space over $R\left[T, T^{-1}\right]$. Then there exist quadratic spaces $q_{1}, q_{2}$ over $R$ and $Q \in \operatorname{Pic} R$ such that

$$
q \perp h^{m} \simeq\left(q_{1} \perp T q_{2} \perp H(Q)\right) \perp h^{m} .
$$

Proof. By Lemma 1.4, $q \perp h^{m} \simeq q_{1}^{\prime} \perp T q_{2}^{\prime} \perp h^{m-1} \perp H(P), P \in$ Pic $R, q_{1}^{\prime}, q_{2}^{\prime}$ quadratic spaces over $R$. Since $q$ is isotropic, Witt index $\left(q_{1}^{\prime} \perp T q_{2}^{\prime} \perp h^{m-1} \perp H(P)\right)$ $\geqslant m+1$ over $K\left[T, T^{-1}\right]$. Thus $q_{1}^{\prime} \perp T q_{2}^{\prime}$ and hence by Lemma $1.1, q_{1}^{\prime}$ or $q_{2}^{\prime}$ is isotropic. Let $q_{1}^{\prime} \perp T q_{2}^{\prime} \simeq q_{1} \perp T q_{2} \perp H(Q), Q \in \operatorname{Pic} R$. Then

$$
q \perp h^{m} \simeq q_{1} \perp T q_{2} \perp h^{m-1} \perp H(P \oplus Q) \simeq q_{1} \perp T q_{2} \perp h^{m} \perp H\left(P \otimes_{R} Q\right) .
$$

COROLlaRY 1.6. Let $R$ be a principal ideal domain. If $q$ is any quadratic space over $R\left[T, T^{-1}\right]$, there exist quadratic spaces $q_{1}$ and $q_{2}$ over $R$ such that $q \perp h^{m} \simeq q_{1} \perp T q_{2}$ $\perp h^{m}$.

Proof. Since Pic $R$ is trivial, the corollary follows from Lemma 1.4.

2. Quadratic spaces over Laurent extensions of complete d.v.r. We begin with the following.

Proposition 2.1. Let $R$ be a p.i.d. and $q$ an isotropic quadratic space over $R\left[T, T^{-1}\right]$. If $q$ is stably extended from $R$, then $q$ is extended from $R$.

Proof. Let $A=R\left[T, T^{-1}\right]$. Since $q$ is stably extended from $R$, there exists a quadratic space $q_{0}$ over $R$ such that $q \perp h^{m} \simeq\left(q_{0} \otimes_{R} A\right) \perp h^{m}$ for some integer $m \geqslant 0$. The ring $R(T)$ obtained from $R[T]$ by inverting all the monic polynomials in $T$ is a p.i.d. In view of [14, Theorem 3.1], we have $q \otimes_{A} R(T) \simeq q_{0} \otimes_{R} R(T)$. Hence there exists a monic $g \in R[T]$ such that $q \otimes_{A} A_{g} \simeq q_{0} \otimes_{R} A_{g}$. Let $g=T^{n} f, n=$ degree $g, f \in R\left[T^{-1}\right]$. Then $T^{-1}$ and $f$ are coprime in $R\left[T^{-1}\right]$ and the quadratic spaces $q$ over $A, q_{0} \otimes_{R} R\left[T^{-1}\right]_{f}$ over $R\left[T^{-1}\right]_{f}$ and an isometry $\varphi: q \otimes_{A} A_{f} \simeq q_{0} \otimes_{R} A_{f}$ define a quadratic space $\tilde{q}$ over $R\left[T^{-1}\right]$ which is isotropic. In view of [9, Theorem 3.2], $\tilde{q}$ is extended from $R$ and hence $q$ is extended from $R$.

The proof above is an adaptation of a proof of Swan [12, Theorem 1.1]. Using a theorem of Suslin and Kopeiko [13] and a cancellation theorem of Roy [11], one can prove the following theorem on the same lines as the above proposition.

THEOREM. Let $q$ be a quadratic space over $R\left[T, T^{-1}\right]$ which is stably extended from a quadratic space $q_{0}$ over $R$. If Witt index of $q_{0} \geqslant \operatorname{dim} R+1$, then $q$ is extended from $R$. 
We note however that Proposition 2.1 is a sharper result for dimension one in view of the extendibility of isotropic quadratic spaces over $R[T], R$ a Dedekind domain.

LEMMA 2.2 Let $R$ be a local domain with maximal ideal $m$ and residue field $k$. Let $q_{1}, q_{2}$ be quadratic spaces over $R$ and let $\psi \in O_{k(T)}\left(\bar{q}_{1} \perp T \bar{q}_{2} \perp h\right)$, bar denoting reduction modulo $\mathrm{m}$. ( $O$ denotes the orthogonal group.) Then there exists $f \in R[T]$, with $f(0)=1$ and $\varphi \in O_{R\left[T, T^{-1}\right]_{f}}\left(q_{1} \perp T q_{2} \perp h\right)$ such that $\bar{\varphi}=\psi$.

Proof. Let $E O\left(\bar{q}_{1} \perp T \bar{q}_{2}, h\right)$ denote the subgroup of $O\left(\bar{q}_{1} \perp T \bar{q}_{2} \perp h\right)$ generated by elementary orthogonal transformations [11]. Then

$$
O_{k(T)}\left(\bar{q}_{1} \perp T \bar{q}_{2} \perp h\right)=E O_{k(T)}\left(\bar{q}_{1} \perp T \bar{q}_{2}, h\right) \cdot O_{k(T)}(h)
$$

and hence there exists $f \in k[T]$ with $f(0)=1$ such that

$$
\psi=\eta \tau, \quad \eta \in E O_{k\left[T, T^{-1}\right]_{f}}\left(\bar{q}_{1} \perp T \bar{q}_{2}, h\right), \tau \in O_{k\left[T, T^{-1}\right]_{f}}(h) .
$$

Then $\tau$ is of the form

$$
\left(\begin{array}{cc}
u & 0 \\
0 & u^{-1}
\end{array}\right) \text { or }\left(\begin{array}{ll}
0 & u \\
u^{-1} & 0
\end{array}\right), \quad u \in U\left(k\left[T, T^{-1}\right]_{f}\right) .
$$

Let $f=\prod f_{i}^{n_{i}}, f_{i}$ irreducible over $k[T]$ and $f_{i}(0)=1$. Let $g_{i} \in R[T]$ be lifts of $f_{i}$ with $g_{i}(0)=1$. Let $g=\prod g_{i}$. The map $R\left[T, T^{-1}\right]_{g} \rightarrow k\left[T, T^{-1}\right]_{f}$ is surjective and induces a surjection also on the corresponding group of units. Hence both $\eta$ and $\tau$ can be lifted to $O_{R\left[T, T^{-1}\right]_{g}}\left(q_{1} \perp T q_{2} \perp h\right)$ and this proves the lemma.

Lemma 2.3. Let $R, \mathfrak{m}, k$ be as in Lemma 2.2. Let $\mathscr{F}$ be a vector bundle over $\mathbf{P}_{1}(R)$

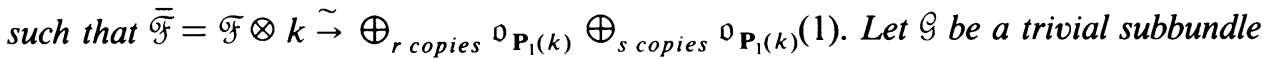
of $\overline{\mathscr{F}}$, which is a direct summand. Then there exists a trivial subbundle $\widetilde{F}_{1}$ of $\mathscr{F}_{\text {which }}$ is a direct summand and such that $\overline{\mathscr{F}}_{1}=\mathcal{G}$.

Proof. Let $i: \mathcal{G} \rightarrow \overline{\mathscr{F}}$ be the inclusion and $p: \overline{\mathscr{F}} \rightarrow \mathcal{G}$ a projection. Since $\operatorname{Hom}(\mathcal{G}, \overline{\mathscr{F}})$ and $\operatorname{Hom}(\overrightarrow{\mathscr{F}}, \mathcal{G})$ are direct sums of copies of $\mathfrak{o}, \mathfrak{o}(1)$ or $\mathfrak{o}(-1)$, it follows that $H^{1}\left(\mathbf{P}_{1}(k), \operatorname{Hom}(\mathcal{G}, \overline{\mathscr{F}})\right)=H^{1}\left(\mathbf{P}_{1}(k), \operatorname{Hom}(\overline{\mathscr{F}}, \mathcal{G})\right)=0$. Let $\mathscr{F}_{1}$ be a trivial bundle over $\mathbf{P}_{1}(R)$ with $\overline{\mathscr{F}}_{1}=\mathcal{G}$. Then the maps $i$ and $p$ can be lifted to $\tilde{i}: \mathscr{F}_{1} \rightarrow \mathscr{F}$ and $\tilde{p}: \mathscr{F} \rightarrow \mathscr{F}_{1}$ $[2,4.6 .2]$. The map $\psi=\tilde{p} \tilde{i}$ is an automorphism of $\mathscr{F}_{1}$, since its reduction modulo $\mathrm{m}$ is identity. Then $j=\psi^{-1} \tilde{i}: \mathscr{F}_{1} \rightarrow \mathscr{F}$ is a direct injection and $\overline{j\left(\mathscr{F}_{1}\right)}=\mathcal{G}$.

THEOREM 2.4. Let $R$ be a complete discrete valuation ring. Any quadratic space over $R\left[T, T^{-1}\right]$ is isometric to $q_{1} \perp T q_{2}, q_{1}, q_{2}$ being quadratic spaces over $R$. In particular, any quadratic space over $R\left[T, T^{-1}\right]$ is an orthogonal sum of rank one quadratic spaces.

Proof. Let $\pi$ be a parameter in $R$ and $k=R /(\pi)$, bar denoting reduction modulo $\pi, K=R_{\pi}=$ quotient field of $R$, and let $A=R\left[T, T^{-1}\right]$. Let $q$ be a quadratic space over $A$. Then by Corollary 1.6, $q$ is stably isometric to $q_{1} \perp T q_{2}, q_{1}, q_{2}$ quadratic spaces over $R$. In view of Lemma 1.3 , over $K\left[T, T^{-1}\right]$ and $k\left[T, T^{-1}\right], q$ and $q_{1} \perp T q_{2}$ are isometric.

Let $q$ be anisotropic. Then $q_{1}$ and $q_{2}$ are anisotropic over $R$ and since $R$ is complete, the reductions $\bar{q}_{1}$ and $\bar{q}_{2}$ modulo $(\pi)$ are anisotropic. Hence, by Lemma 
1.1, $\bar{q}_{1} \perp T \bar{q}_{2}$ is anisotropic. Since $\bar{q} \stackrel{\sim}{\rightarrow} \bar{q}_{1} \perp T \bar{q}_{2}, \bar{q}$ is also anisotropic. In view of [5, Proposition 1.1] any isometry $\varphi: q \otimes_{A} K\left[T, T^{-1}\right] \simeq\left(q_{1} \perp T q_{2}\right) \otimes_{A} K\left[T, T^{-1}\right]$ is defined over $A$ and hence $q \simeq q_{1} \perp T q_{2}$ over $A$.

Let now $q$ be isotropic. Then by Lemma $1.1, q_{1}$ or $q_{2}$ is isotropic and hence splits off an $h$. We assume that $q$ is stably isometric to $q_{1} \perp T q_{2} \perp h$, where $q_{1}$ and $q_{2}$ are quadratic spaces over $R$. Since all projective modules over $R\left[T, T^{-1}\right]$ are free, we identify isometry classes of quadratic forms with equivalence classes of symmetric matrices. Let $\beta_{1}$ be the matrix representing $q_{1}$, and $\beta_{2}$ the matrix representing $q_{2}$ (over $R$ ). Then the matrix

$$
\alpha_{0}=\left(\begin{array}{lll}
\beta_{1} & & \\
& T \beta_{2} & \\
& & h
\end{array}\right)
$$

represents $q_{1} \perp T q_{2} \perp h$, where $h=\left(\begin{array}{ll}0 & 1 \\ 1 & 0\end{array}\right)$. Let $\alpha$ be the matrix of $q$. We show that $\alpha \sim \alpha_{0}$, under the orthogonal equivalence of matrices. By Lemma 1.3, there exists $V \in \mathrm{GL}_{N}\left(k\left[T, T^{-1}\right]\right)$ such that $V \overline{\boldsymbol{\alpha}} V^{t}=\bar{\alpha}_{0}$, where $N=n+m+2, n=\operatorname{rank} q_{1}$, $m=\operatorname{rank} q_{2}$. Since the map $\mathrm{GL}_{N}\left(R\left[T, T^{-1}\right]\right) \rightarrow \mathrm{GL}_{N}\left(k\left[T, T^{-1}\right]\right)$ is surjective, $k\left[T, T^{-1}\right]$ being Euclidean, there exists $U \in \mathrm{GL}_{N}(A)$ such that $\bar{U}=V$. Replacing $\alpha$ by $U \alpha U^{t}$, we assume that $\bar{\alpha}=\bar{\alpha}_{0}$. Since $R\left(T^{-1}\right.$ ) (the ring obtained by inverting monics in $\left.R\left[T^{-1}\right]\right)$ is a p.i.d., by [14, Theorem 3.1], $\alpha$ and $\alpha_{0}$ are equivalent over $R\left(T^{-1}\right)$. Hence there exists $f \in R[T]$ with $f(0)=1$ such that $\alpha \sim \alpha_{0}$ over $A_{f}$. Let $W \alpha W^{t}=\alpha_{0}, W \in \mathrm{GL}_{N}\left(A_{f}\right)$. Then $\bar{W} \in O_{k\left[T, T^{-1}\right]_{f}^{-}}\left(\bar{\alpha}_{0}\right)$ and by Lemma 2.2 , there exists $f^{\prime} \in R[T]$ with $f^{\prime}(0)=1$ and $X \in O_{R\left[T, T^{-1}\right]_{f^{\prime}}}\left(\alpha_{0}\right)$ such that $\bar{X}=\bar{W}$. Replacing $f$ by $f f^{\prime}$ and denoting it again by $f$, we have $\left(X^{-1} W\right) \alpha \cdot\left(X^{-1} W\right)^{t}=\alpha_{0}$ with $X^{-1} W \in \mathrm{GL}_{N}\left(R\left[T, T^{-1}\right]_{f}\right)$ and $\overline{X^{-1} W}=$ Identity. Let $\tilde{\alpha}$ be the symmetric matrix over $R[T]$ obtained by taking $\alpha$ over $R\left[T, T^{-1}\right], \alpha_{0}$ over $R[T]_{f}$ and patching them over $R\left[T, T^{-1}\right]_{f}$ by $Z=X^{-1} W$ (noting that $T$ and $f$ are coprime in $R[T]$ ). Then $\overline{\tilde{\alpha}}$ is obtained by patching $\bar{\alpha}_{0}$ over $k\left[T, T^{-1}\right]$ and $k[T]_{f}^{-}$by $\bar{Z}=$ Identity over $k\left[T, T^{-1}\right]_{f}$. Hence there exists $\bar{Y} \in \mathrm{GL}_{N}(k[T])$ such that $\bar{Y} \overline{\tilde{\alpha}} \bar{Y}^{t}=\bar{\alpha}_{0}$. Let $Y \in$ $\mathrm{GL}_{N}(R[T])$ be a lift of $\bar{Y}$. We replace $\tilde{\alpha}$ by $Y \tilde{\alpha} Y^{t}$ and assume that $\overline{\tilde{\alpha}}=\bar{\alpha}_{0}$.

Since $\tilde{\alpha} \sim \alpha$ over $R\left[T, T^{-1}\right]$, over $R(T)$, which is a p.i.d., we have $\tilde{\alpha} \sim \alpha_{0}$. Hence there exists $f \in R\left[T^{-1}\right]$ with $f(0)=1$ and $B \in \mathrm{GL}_{n}\left(A_{f}\right)$ with $B \tilde{\alpha} B^{t}=\alpha_{0}$. Using similar arguments as above, by changing $f$ suitably, we assume that $\bar{B}=$ Identity. Let

$$
\alpha_{0}^{\prime}=\left(\begin{array}{ccc}
\beta_{1} & & \\
& T^{-1} \beta_{2} & \\
& & h
\end{array}\right) .
$$

We define a vector bundle $\mathscr{F}$ over $\mathbf{P}_{1}(R)$ with a quadratic structure (not necessarily nonsingular) as follows: $\tilde{\alpha}$ over Spec $R[T], \alpha_{0}^{\prime}$ over $\operatorname{Spec} R\left[T^{-1}\right]_{f}$ and the matrix

$$
\left(\begin{array}{ccc}
I_{n} & & \\
& T^{-1} I_{m} & \\
& & I_{2}
\end{array}\right) \cdot B
$$


over Spec $A_{f}$ as a patching isometry. The bundle $\overline{\mathscr{F}}$ is given by the patching matrix ( over $k\left[T, T^{-1}\right]_{f}^{-}$)

$$
\left(\begin{array}{lll}
I_{n} & & \\
& T^{-1} I_{m} & \\
& & I_{2}
\end{array}\right)
$$

and hence $\overline{\mathscr{F}}$ is isometric to $\overline{\mathscr{F}_{1}} \perp \overline{\mathscr{F}}_{2} \perp \overline{\mathscr{F}}_{3}$ where $\overline{\mathscr{F}_{1}}=\bigoplus_{n \text { copies }} \mathfrak{o}, \overline{\mathscr{F}}_{2}=\bigoplus_{m \text { copies }} \mathfrak{o}(1)$, $\overline{\mathscr{F}}_{3}=\oplus_{2 \text { copies }} \mathfrak{D}$ and the quadratic form on $\overline{\mathscr{F}}$ restricted to $\overline{\mathscr{F}}_{1}$ is given by the matrix $\bar{\beta}_{1}$, the quadratic structure on $\overline{\mathscr{F}}_{2}$ being given by $\left(T \bar{\beta}_{2}, T^{-1}, T^{-1} \bar{\beta}_{2}\right)$ and $\overline{\mathscr{F}}_{3} \stackrel{\sim}{\rightarrow} H(\mathfrak{o})$. By Lemma 2.3, $\mathscr{F}$ contains a trivial subbundle $\mathscr{F}_{1}$ of rank $n$ which is a direct summand such that its reduction modulo $\pi$ is $\overline{\mathscr{F}}_{1}$. Since the bundle $\mathscr{F}_{1}$ is trivial, the quadratic form on $\mathscr{F}$, restricted to $\mathscr{F}_{1}$, is extended from a form $\beta_{1}^{\prime}$ over $R$ and its reduction modulo $\pi$ is isometric to $\bar{\beta}_{1}$. Since $R$ is complete, and $\bar{\beta}_{1}^{\prime} \simeq \bar{\beta}_{1}$, we have $\beta_{1}^{\prime} \simeq \beta_{1}$. Hence $\left(\mathscr{F}_{1}, \beta_{1}\right)$ splits off an orthogonal summand of $\mathscr{F}$. Restricting to Spec $R[T]$, we see that $\tilde{\alpha}$ contains an orthogonal summand isometric to $\beta_{1}$. Since $\tilde{\alpha} \sim \alpha$ over $R\left[T, T^{-1}\right]$, it follows that $\alpha$ splits off an orthogonal summand isometric to $\beta_{1}$. Let

$$
\alpha=\left(\begin{array}{cc}
\beta_{1} & 0 \\
0 & \alpha^{\prime}
\end{array}\right)
$$

Then $\alpha^{\prime}$ is stably isometric to

$$
\left(\begin{array}{ll}
T \beta_{2} & \\
& h
\end{array}\right) .
$$

Hence $T^{-1} \alpha^{\prime}$ is stably extended from

$$
\left(\begin{array}{ll}
\beta_{2} & \\
& h
\end{array}\right)
$$

and by Proposition 2.1, extended from

$$
\left(\begin{array}{ll}
\beta_{2} & \\
& h
\end{array}\right) \text {. }
$$

Thus

$$
\alpha \sim\left(\begin{array}{lll}
\beta_{1} & & \\
& T \beta_{2} & \\
& & h
\end{array}\right)
$$

and this completes the proof of the theorem.

3. Quadratic spaces over Laurent extensions of Dedekind domains. Let $R \hookrightarrow S$ be integral domains and $h \in R$ be a nonzero element of $R$. Let the natural map $R / h R \rightarrow S / h S$ be an isomorphism. We call the following cartesian square a patching diagram:

$$
\begin{array}{lll}
R & \hookrightarrow & S \\
\eta & & \eta \\
R_{h} & \hookrightarrow & S_{h}
\end{array}
$$


Let $\Re$ denote the category of quadratic spaces over $R$ and $\Re$ the category whose objects are triples $\left(q_{1}, \varphi, q_{2}\right)$ where $q_{1}$ is a quadratic space over $S, q_{2}$ a quadratic space over $R_{h}$ and $\varphi: q_{1} \otimes_{S} S_{h} \stackrel{\sim}{\rightarrow} q_{2} \otimes_{R_{h}} S_{h}$ an isometry, with obvious morphisms of triples. We have a natural functor $T: \Re \rightarrow \Re$ with $T(q)=\left(q \otimes_{R} S, \operatorname{Id}, q \otimes_{R} R_{h}\right)$. In view of [6, Theorem 1], $T$ is an equivalence of categories.

LEMMA 3.1. Let $P$ be a finitely-generated projective $R$-module. For $\alpha(T) \in$ End $_{S_{h}[T]}\left(P \otimes_{R} S_{h}[T]\right)$ with $\alpha(0)=$ Identity, there exists an integer $N \geqslant 0$ such that for $n \geqslant N, \alpha\left(h^{n} T\right) \in \operatorname{End}_{S[T]}\left(P \otimes_{R} S[T]\right)$.

Proof. Let

$$
\alpha(T)=1+\alpha_{1} T+\cdots+\alpha_{m} T^{m}, \quad \alpha_{i} \in \operatorname{End}_{S_{h}}\left(P \otimes_{R} S_{h}\right) .
$$

Since $P$ is finitely generated, there exists an integer $N$ such that for $n \geqslant N$, $h^{n} \alpha_{i} \in \operatorname{End}_{S}\left(P \otimes_{R} S\right)$ for $1 \leqslant i \leqslant m$. Then $\alpha\left(h^{n} T\right) \in \operatorname{End}_{S[T]}\left(P \otimes_{R} S[T]\right)$.

LEMMA 3.2. Let $(P, q)$ be a quadratic space over $R$. Then, for

$$
\alpha(T) \in O_{S_{h}[T]}\left(q \otimes_{R} S_{h}[T]\right) \text { with } \alpha(0)=\text { Identity, }
$$

there exists $N \geqslant 0$ such that $\alpha\left(h^{n} T\right) \in O_{S[T]}(q \otimes S[T]), n \geqslant N$.

Proof. We have $O_{S_{h}[T]}\left(q \otimes_{R} S_{h}[T]\right) \cap \operatorname{End}_{S[T]}\left(P \otimes_{R} S[T]\right)=O_{S[T]}\left(q \otimes_{R} S[T]\right)$.

LemMA 3.3. Let $(P, q)$ be a quadratic space over $R$ and $Q \in \operatorname{Pic} R$. Let $E O(q, H(Q))$ denote the elementary orthogonal subgroup of $O(q \perp H(Q))$. Then given $\sigma \in$ $E O_{S_{h}}(q, H(Q))$ and $\tau \in O_{S_{h}}(H(Q))$, there exists $\sigma_{1} \in O_{S}(q \perp H(Q))$ and $\sigma_{2} \in$ $O_{R_{h}}(q \perp H(Q))$ such that $\tau \sigma=\sigma_{1} \tau \sigma_{2}$.

Proof. Let $\alpha_{i}, 1 \leqslant i \leqslant l$ (resp. $\beta_{j}, l+1 \leqslant j \leqslant l+s$ ), be a set of generators of $\operatorname{Hom}(P, Q)\left(\operatorname{resp} . \operatorname{Hom}\left(P, Q^{*}\right)\right)$. Let $e_{\lambda}^{k}=E_{\lambda \alpha_{k}}$ for $1 \leqslant k \leqslant l$ and $e_{\lambda}^{k}=E_{\lambda \beta_{k}}^{*}$, for $l+1 \leqslant k \leqslant l+s, \lambda \in S_{h}$, defined in [11, pp. 292, 293]. Then $E O_{S_{h}}(q, H(Q))$ is the subgroup generated by $e_{\lambda}^{k}, \lambda \in S_{h}, 1 \leqslant k \leqslant l+m$. Let $\sigma=\prod_{k=1}^{m} e_{\lambda_{k}}^{i_{k}}$ and let $\sigma_{p}=$ $\prod_{k=1}^{p-1} e_{\lambda_{k}}^{i_{k}}, 1 \leqslant p \leqslant m$. Then by Lemma 3.2, there exists an integer $N$ such that $\left(\tau \sigma_{p}\right) \cdot e_{h^{N} T}^{i_{p}} \cdot\left(\tau \sigma_{p}\right)^{-1} \in O_{S[T]}(q \perp H(Q))$ for $1 \leqslant p \leqslant m$. Since $S h^{N}+R=S$, there exist $\mu_{k} \in S, \nu_{k} \in R_{h}$ such that $\lambda_{k}=h^{N} \mu_{k}+\nu_{k}, 1 \leqslant k \leqslant m$. Then specialising $T=\mu_{p} \in S$, we have,

$$
\left(\tau \sigma_{p}\right) \cdot e_{h^{N} \cdot \mu_{p}}^{i_{p_{p}}} \cdot\left(\tau \sigma_{p}\right)^{-1} \in O_{S}(q \perp H(Q)) \text { for } 1 \leqslant p \leqslant m .
$$

We have

$$
\begin{aligned}
\tau \boldsymbol{\sigma} & =\tau \cdot \prod_{k=1}^{m} e_{\lambda_{k}}^{i_{k}}=\tau \cdot \prod_{k=1}^{m} e_{h^{N} \cdot \mu_{k}}^{i_{k}} \cdot e_{\nu_{k}}^{i_{k}}=\tau \cdot \prod_{k=m}^{1}\left(\sigma_{k} \cdot e_{h^{N} \mu_{k}}^{i_{k}} \sigma_{k}^{-1}\right) \cdot \prod_{k=1}^{m} e_{\nu_{k}}^{i_{k}} \\
& =\prod_{k=m}^{1} \tau \sigma_{k} \cdot e_{h^{N} \mu_{k}}^{i_{k}}\left(\tau \sigma_{k}\right)^{-1} \cdot \tau \cdot \prod_{k=1}^{m} e_{\nu_{k}}^{i_{k}}=\sigma_{1} \cdot \tau \cdot \sigma_{2}
\end{aligned}
$$

where $\sigma_{1} \in O_{S}(q \perp H(Q)), \sigma_{2} \in O_{R_{h}}(q \perp H(Q))$.

Remark. The idea of the proof of the above lemma is due to Suslin. 
Proposition 3.4. Let $R$ be a principal ideal domain. Then every isotropic quadratic space over $R\left[T, T^{-1}\right]$ is isometric to $q_{1} \perp T q_{2}, q_{1}, q_{2}$ quadratic spaces over $R$.

Proof. By Corollary $1.5, q$ is stably isometric to $q_{1} \perp T q_{2} \perp h, q_{1}, q_{2}$ quadratic spaces over $R$. Let $K$ be the quotient field of $R$. Over $K\left[T, T^{-1}\right]$, by Lemma 1.3, $q \simeq q_{1} \perp T q_{2} \perp h$. We assume, without loss of generality, that by inverting a prime $p \in R, q \stackrel{\sim}{\rightarrow} q_{1} \perp T q_{2} \perp h$. Let $\hat{R}$ denote the completion of $R$ at the prime ideal $(p)$. We have a patching diagram:

$$
\begin{array}{ccc}
R\left[T, T^{-1}\right] & \hookrightarrow & \hat{R}\left[T, T^{-1}\right] \\
\eta & & \eta \\
R_{p}\left[T, T^{-1}\right] & \hookrightarrow & \hat{R}_{p}\left[T, T^{-1}\right]
\end{array}
$$

Since $\hat{R}$ is a complete d.v.r., we have an isometry $\varphi: \underset{q}{\sim} q_{1} \perp T q_{2} \perp h$ over $\hat{R}\left[T, T^{-1}\right]$. By assumption, there exists an isometry $\psi: \underset{q}{\sim} \stackrel{\sim}{\rightarrow} q_{1} \perp T q_{2} \perp h$ over $R_{p}\left[T, T^{-1}\right]$. If $\varphi$ and $\psi$ coincide over $\hat{R}_{p}\left[T, T^{-1}\right]$, then they define an isometry

$$
q \simeq q_{1} \perp T q_{2} \perp h
$$

over $R\left[T, T^{-1}\right]$. Otherwise, $\varphi \psi^{-1} \in O_{\hat{R}_{p}\left[T, T^{-1}\right]}\left(q_{1} \perp T q_{2} \perp h\right)$. Since $\hat{R}_{p}$ is a field, in view of [10, Lemma 1.3], there exist $\eta \in E O_{\hat{R}_{p}\left[T, T^{-1}\right]}\left(q_{1} \perp T q_{2}, h\right)$ and $\tau \in$ $O_{\hat{R}_{p}\left[T, T^{-1}\right]}(h)$ such that $\varphi \psi^{-1}=\tau \eta$. By Lemma 3.3,

$$
\tau \eta=\eta_{1} \tau \eta_{2}, \quad \eta_{1} \in O_{\hat{R}\left[T, T^{-1}\right]}\left(q_{1} \perp T q_{2} \perp h\right), \eta_{2} \in O_{R_{p}\left[T, T^{-1}\right]}\left(q_{1} \perp T q_{2} \perp h\right) .
$$

The element $\tau$ is of the form

$$
\left(\begin{array}{cc}
u & 0 \\
0 & u^{-1}
\end{array}\right) \text { or }\left(\begin{array}{ll}
0 & u \\
u^{-1} & 0
\end{array}\right), \quad u \in U\left(\hat{R}_{p}\left[T, T^{-1}\right]\right) .
$$

Since $u=u_{1} u_{2}, u_{1} \in U\left(\hat{R}\left[T, T^{-1}\right]\right), u_{2} \in U\left(R_{p}\left[T, T^{-1}\right]\right), \tau=\tau_{1} \tau_{2}, \tau_{1} \in O_{\hat{R}\left[T, T^{-1}\right]}(h)$, $\tau_{2} \in O_{R_{p}\left[T, T^{-1}\right]}(h)$. Hence $\tau \eta=\eta_{1} \tau_{1} \cdot \tau_{2} \eta_{2}=\sigma_{1} \cdot \sigma_{2}$, where $\sigma_{1}=\eta_{1} \tau_{1}, \sigma_{2}=\tau_{2} \eta_{2}$. Replacing $\varphi$ and $\psi$ by $\varphi^{\prime}=\left(\eta_{1} \tau_{1}\right)^{-1} \varphi, \psi^{\prime}=\tau_{2} \eta_{2} \cdot \psi$, we have, $\varphi^{\prime}=\psi^{\prime}$ over $\hat{R}_{p}\left[T, T^{-1}\right]$ and hence define an isometry $q \simeq q_{1} \perp T q_{2} \perp h$ over $R\left[T, T^{-1}\right]$.

THEOREM 3.5. Let $R$ be a Dedekind domain and $q$ an isotropic quadratic space over $R\left[T, T^{-1}\right]$. Then $q \simeq q_{1} \perp T q_{2}, q_{1}$ and $q_{2}$ being quadratic spaces over $R$.

Proof. By Corollary 1.5, $q$ is stably isometric to $q_{1} \perp T q_{2} \perp H(Q), q_{1}, q_{2}$ quadratic spaces over $R$ and $Q \in \operatorname{Pic} R$. Let $K$ denote the quotient field of $R$. Then by Lemma 1.3, $q \simeq q_{1} \perp T q_{2} \perp H(Q)$ over $K\left[T, T^{-1}\right]$. Thus, there exists $\lambda \in R$, $\lambda \neq 0$, such that there is an isometry $\psi: q \otimes R_{\lambda}\left[T, T^{-1}\right] \simeq\left(q_{1} \perp T q_{2} \perp H(Q)\right) \otimes$ $R_{\lambda}\left[T, T^{-1}\right]$. Let $(\lambda)=\prod_{i=1}^{r} \wp_{i}^{n_{i}}, \wp_{i} \in$ Spec $R$ and let $S$ denote the semilocalisation of $R$ at the set $\left\{\wp_{i}\right\}_{i=1}^{r}$ of prime ideals of $R$. Then we have a patching diagram:

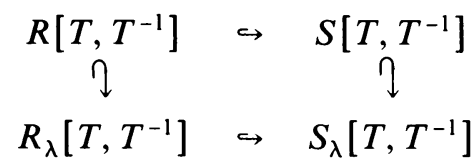

Since $S$ is a semilocal domain of dimension one, $S$ is p.i.d. and by Proposition 3.4, we have an isometry $\varphi: q \otimes_{R} S \stackrel{\sim}{\rightarrow}\left(q_{1} \perp T q_{2} \perp H(Q)\right) \otimes_{R} S$. If $\varphi$ and $\psi$ coincide over 
$S_{\lambda}\left[T, T^{-1}\right]$, they define an isometry $q \stackrel{\sim}{\rightarrow} q_{1} \perp T q_{2} \perp H(Q)$ over $R\left[T, T^{-1}\right]$. Otherwise, $\varphi \psi^{-1} \in O_{S_{\lambda}\left[T, T^{-1}\right]}\left(q_{1} \perp T q_{2} \perp H(Q)\right)$. Since $\lambda \in \operatorname{rad} S, S_{\lambda}$ is a field and by [10, Lemma 1.3],

$$
\varphi \psi^{-1}=\tau \eta, \quad \tau \in O_{S_{\lambda}\left[T, T^{-1}\right]}(H(Q)), \eta \in E O_{S_{\lambda}\left[T, T^{-1}\right]}\left(q_{1} \perp T q_{2}, H(Q)\right) .
$$

By Lemma 3.3, there exist

$$
\eta_{1} \in O_{S\left[T, T^{-1}\right]}\left(q_{1} \perp T q_{2} \perp H(Q)\right), \quad \eta_{2} \in O_{R_{\lambda}\left[T, T^{-1}\right]}\left(q_{1} \perp T q_{2} \perp H(Q)\right)
$$

such that $\tau \eta=\eta_{1} \tau \eta_{2}$. Modifying $\varphi$ and $\psi$ by $\eta_{1}$ and $\eta_{2}$ respectively, we may assume that $\varphi \psi^{-1}=\tau$. Then identifying quadratic spaces over $R$ with triples in $\Re$,

$$
q \simeq\left(q_{1} \perp T q_{2} \perp H(Q), \mathrm{Id} \perp \tau, q_{1} \perp T q_{2} \perp H(Q)\right) \simeq q_{1} \perp T q_{2} \perp q_{3}
$$

where $q_{3} \simeq(H(Q), \tau, H(Q))$. Since discriminant of $q_{3}$ is locally -1 , disc $q_{3}=-1$ and in view of [1, Proposition 5.1], $q_{3} \simeq H\left(Q^{\prime}\right), Q^{\prime} \in \operatorname{Pic} R\left[T, T^{-1}\right]=\operatorname{Pic} R$. Thus $q \simeq$ $\left(q_{1} \perp H\left(Q^{\prime}\right)\right) \perp T q_{2}$ and this completes the proof of the theorem.

REMARK 3.6. Theorem 3.5 is false for anisotropic quadratic spaces over Laurent extensions of Dedekind domains. Let $q$ be the rank 4 quadratic space over $\mathbf{R}[X, Y]$ given by the reduced norm on the nonfree projective ideal $P$ of $\mathbf{H}[X, Y]$ defined in [7] as the kernel of the surjective homomorphism

$$
\begin{array}{ccc}
\mathbf{H}[X, Y]^{2} & \rightarrow & \mathbf{H}[X, Y], \\
(1,0) & \mapsto & X+i, \\
(0,1) & \mapsto & Y+j .
\end{array}
$$

In fact $q$ is stably isometric to $\langle 1,1,1,1\rangle$, but not extended from $\mathbf{R}[X]$ and in fact over $\mathbf{R}[X]_{\left(1+x^{2}\right)}[Y], q$ remains nonextended from $\mathbf{R}[X]_{\left(1+x^{2}\right)}[\mathbf{8}$, Theorem 2.1]. Let $R=\mathbf{R}[X]_{\left(1+x^{2}\right)}$ and let $\tilde{q}=q \otimes_{\mathbf{R}[X, Y]} R\left[Y, Y^{-1}\right]$. Suppose $\tilde{q} \stackrel{\sim}{\rightarrow} q_{1} \perp T q_{2}, q_{1}, q_{2}$ quadratic spaces over $R$. Let $K$ be the quotient field of $R$. Then, $\tilde{q}$ is stably isometric to $\langle 1,1,1,1\rangle$ and hence over $K\left[Y, Y^{-1}\right], q_{1} \perp T q_{2} \simeq\langle 1,1,1,1\rangle$. Since these forms are anisotropic, it follows that $q_{2}=0$. Thus, $\tilde{q}$ is stably extended from $R$. In this case, it should be extended from $\tilde{q}(1) \stackrel{\sim}{\rightarrow}\langle 1,1,1,1\rangle$. Suppose $\varphi: \tilde{q} \stackrel{\sim}{\rightarrow}\langle 1,1,1,1\rangle$ is an isometry over $R\left[Y, Y^{-1}\right]$. Since modulo $Y, q$ and $\langle 1,1,1,1\rangle$ are anisotropic, in view of [5, Proposition 1.1], $\varphi$ is defined over $R[Y]$ contradicting that over $R[Y], q \neq$ $\langle 1,1,1,1\rangle$.

\section{REFERENCES}

1. H. Bass, Modules which support nonsingular forms, J. Algebra 13 (1969), 246-252.

2. A. Grothendieck, Eléments de géométrie algébrique, Inst. Haute Études Sci. Publ. Math. 11 (1961).

3. M. Karoubi, Localisation de formes quadratiques. II, Ann. Sci. École Norm. Sup. (4) 8 (1975), 99-155.

4. M. Knebusch, Grothendieck- und Wittringe von nichtausgearteten symmetrischen Bilnearformen, S. B. Heidelberger Akad. Wiss. Math.-Natur. Kl. 1969/70, 93-157.

5. M. A. Knus, R. Parimala and R. Sridharan, Non-free projective modules over $\mathbf{H}[X, Y]$ and stable bundles over $\mathbf{P}_{\mathbf{2}}(\mathbf{C})$, Invent. Math. 65 (1981), 13-27.

6. M. Ojanguren, Quadratic forms over regular rings, J. Indian Math. Soc. 44 (1979), 109-116.

7. M. Ojanguren and R. Sridharan, Cancellation of Azumaya algebras, J. Algebra 18 (1971), 501-505.

8. S. Parimala, Failure of a quadratic analogue of Serre's conjecture, Amer. J. Math. 100 (1978), 913-924. 
9. R. Parimala, Quadratic forms over polynomial rings over Dedekind domains, Amer. J. Math. 103 (1981), 289-296.

10. Parvin Sinclair, Structure and cancellation of quadratic spaces over Laurent extensions of rings of dimension one, preprint.

11. A. Roy, Cancellation of quadratic forms over commutative rings, J. Algebra 10 (1968), 286-298.

12. R. G. Swan, Projective modules over Laurent polynomial rings, Trans. Amer. Math. Soc. 237 (1978), $111-120$.

13. A. Suslin and V. I. Kopeiko, Modules quadratiques et groupes orthogonaux sur les anneaux de pôlynomes, Zap. Naučn. Sem. Leningrad. Otdel. Mat. Inst. Steklov. 71 (1977), 216-250.

14. R. C. Wagner, Some Witt cancellation theorems, Amer. J. Math. 94 (1972), 206-220.

School of Mathematics, Tata Institute of Fundamental Research, Bombay 400 005, India 